\title{
Section E6.1-6.4 of the ACMG technical standards and guidelines: chromosome studies of neoplastic blood and bone marrow-acquired chromosomal abnormalities
}

\author{
Fady M. Mikhail, MD, PhD ${ }^{1}$, Nyla A. Heerema, $\mathrm{PhD}^{2}$, Kathleen W. Rao, PhD, ${ }^{3,4,8}$, Rachel D. Burnside, PhD \\ Athena M. Cherry, $\mathrm{PhD}^{6}$ and Linda D. Cooley, MD, MBA7; on behalf of the American College of Medical \\ Genetics and Genomics (ACMG) Laboratory Quality Assurance Committee
}

\begin{abstract}
Disclaimer: These American College of Medical Genetics and Genomics standards and guidelines are developed primarily as an educational resource for clinical laboratory geneticists to help them provide quality clinical laboratory genetic services. Adherence to these standards and guidelines is voluntary and does not necessarily ensure a successful medical outcome. These standards and guidelines should not be considered inclusive of all proper procedures and tests or exclusive of other procedures and tests that are reasonably directed to obtaining the same results. In determining the propriety of any specific procedure or test, the clinical laboratory geneticist should apply his or her own professional judgment to the specific circumstances presented by the individual patient or specimen. Clinical

laboratory geneticists are encouraged to document in the patient's record the rationale for the use of a particular procedure or test, whether or not it is in conformance with these standards and guidelines. They also are advised to take notice of the date any particular guideline was adopted, and to consider other relevant medical and scientific information that becomes available after that date. It also would be prudent to consider whether intellectual property interests may restrict the performance of certain tests and other procedures.
\end{abstract}

Cytogenetic analyses of hematological neoplasms are performed to detect and characterize clonal chromosomal abnormalities that have important diagnostic, prognostic, and therapeutic implications. At the time of diagnosis, cytogenetic abnormalities assist in the diagnosis of such disorders and can provide important prognostic information. At the time of relapse, cytogenetic analysis can be used to confirm recurrence of the original neoplasm, detect clonal disease evolution, or uncover a new unrelated neoplastic process. This section deals specifically with the standards and guidelines applicable to chromosome studies of neoplastic blood and bone marrow-acquired chromosomal abnormalities.
This updated Section E6.1-6.4 has been incorporated into and supersedes the previous Section E6 in Section E: Clinical Cytogenetics of the 2009 Edition (Revised 01/2010), American College of Medical Genetics and Genomics Standards and Guidelines for Clinical Genetics Laboratories.

Genet Med advance online publication 28 April 2016

Key Words: bone marrow; cancer cytogenetics; clonal chromosomal abnormalities; cytogenetic analysis; hematological malignancies

\subsection{GENERAL CONSIDERATIONS}

6.1.1 Cytogenetic analyses of neoplastic blood and/or bone marrow-acquired clonal chromosomal abnormalities have been increasingly important in the clinical management of patients with hematological neoplasms. At time of diagnosis, cytogenetic abnormalities assist in the diagnosis of such disorders and can provide important prognostic information. ${ }^{1}$ Furthermore, cytogenetic analysis can provide crucial information regarding specific genetically defined subtypes of these neoplasms that have targeted therapies. At time of relapse, cytogenetic analysis can be used to confirm recurrence of the original neoplasm, detect clonal disease evolution, or uncover a new unrelated neoplastic process.

6.1.2 These cytogenetic analyses include conventional G-banded chromosome analysis, fluorescence in situ hybridization (FISH), and/or chromosomal microarray (CMA). Laboratories should work closely with oncologists and pathologists to determine the order of testing required to obtain relevant cytogenetic information in a cost-effective manner.

6.1.3 Laboratories offering cytogenetic analyses for hematological neoplasms should be familiar with the various chromosomal abnormalities associated with the different neoplasms and their clinical significance. The laboratory should be able to provide a robust analytical and interpretative service for the various hematological neoplasms. All results should be, to the extent possible, interpreted in the context of the clinical, pathologic, and molecular findings. ${ }^{1,2}$

6.1.4 Tissue processing, analytical variables, and turnaround time (TAT) should be determined by the laboratory based on

${ }^{1}$ Department of Genetics, University of Alabama at Birmingham, Birmingham, Alabama, USA; ${ }^{2}$ Department of Pathology, The Ohio State University, Columbus, Ohio, USA; ${ }^{3}$ Department of Pediatrics, University of North Carolina, Chapel Hill, North Carolina, USA; ${ }^{4}$ Department of Pathology, University of North Carolina, Chapel Hill, North Carolina, USA; ${ }^{5}$ Laboratory Corporation of America Holdings, Center for Molecular Biology and Pathology, Research Triangle Park, North Carolina, USA; ${ }^{6}$ Department of Pathology, Stanford University School of Medicine, Stanford, California, USA; ${ }^{7}$ Department of Pathology and Laboratory Medicine, Children's Mercy Hospital, University of Missouri Kansas City Medical School, Kansas City, Missouri, USA. ${ }^{8}$ Deceased. Correspondence: Fady M. Mikhail (fmikhail@uab.edu)

These technical standards and guidelines were approved by the ACMG Board of Directors on 25 January 2016.

Submitted 1 March 2016; accepted 1 March 2016; advance online publication 28 April 2016. doi:10.1038/gim.2016.50 
the indication for cytogenetic referral (e.g., initial diagnosis versus follow-up studies, pre- versus posttransplant studies, and lymphoid versus myeloid malignancies) and the clinical application of the cytogenetic results (e.g., selection of therapy).

6.1.5 Molecular genetics analyses are essential for diagnosis of some hematological neoplasms, and several molecular mutations, not detectable by cytogenetic analyses, provide important diagnostic and prognostic information. These are outside the scope of the current guidelines.

6.1.6 For quality assurance, the laboratory should monitor the numbers and types of hematological neoplasms received, percentage of cases with abnormal results, cell culture success rate, success rate of FISH and CMA studies, TAT, and correlation of FISH and CMA data with G-banded chromosome analysis results.

\subsection{SPECIMEN COLLECTION AND PROCESSING}

\subsubsection{Specimen collection}

6.2.1.1 Only those cells involved in the neoplastic process will harbor the abnormalities being sought. Therefore, the specimen type and culture techniques utilized should optimize the probability of detecting an abnormal clone.

6.2.1.2 In most cases, bone marrow is the tissue of choice for cytogenetic analyses of suspected hematological neoplasms. In some circumstances, alternative specimens may be used, including the following:

a. Peripheral blood specimens may yield informative results when the circulating blast cell percentage is higher than $10 \%$. In general, the abnormal clone can be identified in such specimens, albeit not as often as in bone marrow. Peripheral blood or bone marrow can be used in chronic lymphocytic leukemia (CLL).

b. Bone marrow core biopsy specimens.

c. Bone marrow smears and core biopsy touch imprints can be used for interphase FISH.

d. Lymph node biopsy material or biopsy material from a suspected lymphoid mass are the preferred tissue in all lymphomas.

e. Cerebrospinal fluid.

f. Extramedullary leukemia (myeloid sarcoma, chloroma) tissue biopsy.

6.2.1.3 Specimens should be collected under sterile conditions in sodium heparin tubes for chromosome and/or FISH analyses. Bone marrow aspirate and biopsy specimens should preferably be collected in an appropriate transport medium tube with sodium heparin. The concentration of sodium heparin should be $\sim 20 \mathrm{U} / \mathrm{ml}$ of specimen (per either bone marrow volume alone or per total volume of bone marrow and transport medium combined). EDTA tubes can be used for procedures that require genomic DNA extraction.

6.2.1.4 The volume of bone marrow available will differ for adults and children. An approximate specimen of 1 to $3 \mathrm{ml}$ should be requested. During specimen procurement, several draws are likely to be withdrawn. Because the first draw is more concentrated with neoplastic immature bone marrow cells, it is recommended that cytogenetics receive the first or second draw whenever possible.

6.2.1.5 Specimens should be received by the laboratory as soon as possible, ideally within 24 hours. Also, it is recommended that specimens be maintained at ambient temperature during transit. Extreme temperatures should be avoided.

6.2.1.6 If the specimen size precludes cell culture and conventional G-banded chromosome analysis, bone marrow smears or core biopsy touch imprints can be used for interphase FISH analysis.

\subsubsection{Specimen processing}

6.2.2.1 The laboratory should process the specimen as soon as possible after it is received. The methods that will be used to analyze the specimen should be determined prior to processing whenever possible. If chromosome analysis is requested, cell culture will be required. If FISH and/or CMA analyses are requested, a portion of the specimen can be used for direct harvest of interphase cells and/or genomic DNA extraction.

6.2.2.2 If a bone marrow core biopsy is obtained, it should be disaggregated to generate a cell suspension. This can be achieved by mechanical mincing and/or enzymatic digestion using collagenase. Culture conditions are the same as those for a bone marrow aspirate.

6.2.2.3 Cell culture conditions should be optimized for the specific hematological neoplasm suspected:

a. Acute leukemias, including acute myeloid leukemia (AML), acute lymphoblastic leukemia (ALL), and acute biphenotypic leukemia: Unstimulated short-term cultures are recommended. If sufficient specimen is received, at least two cultures should be initiated, including direct, overnight, and/or 24-hour cultures. In pediatric ALL, an additional unstimulated 48-hour culture can be useful in characterizing the abnormal karyotype. The seeding density is usually 1 to 3 million cells per $\mathrm{ml}$ of medium.

b. Myelodysplastic syndromes (MDS) and myeloproliferative neoplasms (MPN): Same as acute leukemias.

c. Plasma cell dyscrasias, including multiple myeloma (MM) and plasma cell leukemia: Unstimulated 24- and 72-hour cultures as well as 120-hour IL-4-stimulated culture are recommended. ${ }^{3}$ For FISH and/or CMA analyses, if the bone marrow plasma cells percentage (as determined by flow cytometry) is below a certain cutoff value, plasma cell separation is recommended to enrich for the $\mathrm{CD}_{138^{+}}$plasma cell fraction..$^{4,5}$ The laboratory needs to establish its cutoff value for plasma cell enrichment.

d. Chronic lymphoproliferative disorders: Depending on the immunophenotype, additional cultures with B- or T-cell mitogens may be helpful. In CLL and other mature B-cell neoplasms, CpG-oligonucleotide cell stimulation 
is recommended and has been shown to enhance the detection of clonal chromosomal abnormalities. ${ }^{6,7}$

e. Well-differentiated T-cell disorders (e.g., T-cell leukemias, T-cell lymphoma, Sézary syndrome, and mycosis fungoides): T-cell mitogens may be helpful.

\subsection{ANALYSIS}

\subsubsection{Conventional G-banded chromosome analysis}

6.3.1.1 Cell selection: metaphase cells should not be selected for analysis solely on the basis of good chromosome morphology. In general, the technologist should select an area of the slide to begin the analysis and then examine metaphase cells as they appear consecutively in the microscope field, only skipping cells for which extremely poor morphology precludes chromosome identification. This technique can also be performed using automated metaphase finders by examining metaphase cells consecutively captured by the system. Sufficient cells should be analyzed or examined to maximize the detection of an abnormal clone and establish the clonality of the abnormality found. For each abnormal clone identified, clonal cells with the best chromosome morphology should be analyzed, captured, and karyotyped to provide the most accurate breakpoint assignment.

When cells are skipped because of poor morphology, it is important to attempt to count the number of chromosomes. This is particularly true for possible hyperdiploid or hypodiploid pediatric ALL and hyperdiploid plasma cell dyscrasias. In addition, attempts should be made to identify possible structural chromosomal abnormalities, particularly if the disease under consideration is associated with a specific recurring abnormality (e.g., the $t(9 ; 22)$ in chronic myeloid leukemia (CML)).

6.3.1.2 Number of cells evaluated: The number of metaphase cells analyzed versus the number of cells counted or scored should be appropriate for the type of the study (e.g., initial diagnosis or follow-up studies) and the purpose of the study (e.g., detection of residual disease or response to therapy, monitoring for clonal evolution, or monitoring of allogeneic transplant engraftment).

6.3.1.3 Initial diagnostic studies:

a. Analysis: Analyze a minimum of 20 cells from unstimulated cultures. For the mature B- and T-cell disorders, a combination of unstimulated and mitogen-stimulated cultures may be appropriate as described. Unstimulated CLL cultures infrequently yield CLL-related clonal chromosomal abnormalities; however, they can reveal MDSrelated clonal abnormities since some of these patients might have co-morbid MDS because of either prior therapy or age-related. Similarly, unstimulated 24-h MM cultures can reveal co-morbid MDS-related clonal abnormalities.

b. Documentation:

- For the abnormal cells:

- If only one abnormal clone is present: two karyotypes.
- If more than one related abnormal clone is present: two karyotypes of the stemline and one of each sideline.

- If unrelated clones are present: two karyotypes for each stemline and one for each associated pertinent sideline.

- In instances when the sideline contains complex abnormalities, two karyotypes of each sideline may be required for better documentation.

- For the normal cells:

- If only normal cells are present: two karyotypes.

- If normal and abnormal cells are present: one karyotype of a normal cell.

6.3.1.4 Follow-up studies of patients who have had a previous cytogenetic study: For the following analytic guidelines, it is assumed that the laboratory has documentation of the patient's previous cytogenetic results. If the study has been performed elsewhere and there is minimal information available, it is recommended that, except for patients seen for the first time posttransplant, the analysis be considered the same as an initial diagnostic workup (see above).

I. Patients who have not undergone allogeneic hematopoietic cell transplantation:

a. Analysis: analyze 20 cells. If all cells are normal, additional cells may be scored for a specific abnormality by G-banding or FISH if pathology is positive for the diagnosis in question. For some patients, follow-up cytogenetic study is ordered to rule out a therapyassociated malignancy (e.g., MDS) rather than disease recurrence.

b. Documentation:

- For cases with both normal and abnormal cells or only abnormal cells:

- One karyotype of a normal cell, if such a karyotype was not documented in a previous study by the laboratory; otherwise, one normal metaphase spread.

- One or two karyotypes from each abnormal clone for a minimum total of two karyotypes.

- For cases with all normal cells:

- Two karyotypes.

II. Patients who have undergone an allogeneic hematopoietic cell transplantation for whom donor versus recipient origin of the cells can be determined (by sex chromosome complement or cytogenetic heteromorphisms):

For studies aimed solely at determining engraftment status, molecular methods and/or interphase FISH (in the case of opposite sex transplant) are more sensitive than G-banded chromosome analysis and are the preferred methodologies. Therefore, in consultation with the referring physician, cancellation of test 
requests for G-banded chromosome analysis for engraftment status should be considered.

During the course of the cytogenetic analysis, it will become evident whether there is chimerism for donor and recipient cells. It is expected that there will be different approaches used by different laboratories to address these studies.

- If only donor cells are present:

a. Analysis: analyze 20 cells.

b. Documentation: document two karyotypes for each cell line. In such cases, one is documenting either the constitutional karyotype (normal or abnormal of the donor) or the rare event of a malignant process arising in a donor cell.

- If donor and recipient cells are present:

a. Analysis: Analyze recipient cells completely for previously identified clonal chromosome abnormalities and any newly acquired abnormalities. In some cases there may be structural chromosomal abnormalities secondary to chromosome breakage or rearrangement induced by the pretransplant conditioning regimen. The laboratory should distinguish clonal from nonclonal changes and determine the significance of new abnormalities as much as possible.

Analyze all recipient cells present out of 20 cells analyzed. Evaluate each recipient cell for the presence of the abnormality present prior to transplantation (i.e., the diagnostic abnormality). Depending on the number of recipient cells present among the initial 20 metaphase cells scored, additional recipient cells may be analyzed completely and/or scored for the presence of the diagnostic abnormality.

Donor cells: analyze two donor cells if donor cells have not been analyzed in previous studies. Otherwise, simply score these cells as being of donor origin and count.

b. Documentation: for the recipient cells: Two karyotypes of the stemline and one of each sideline. For the donor cells: If donor cells have been documented previously, then provide a single metaphase spread. If donor cells have not been documented previously, then provide two karyotypes.

- If only recipient cells are present:

a. Analysis: analyze 20 cells following the guidelines set forth above with respect to the characterization of secondary abnormalities.

b. Documentation: same as noted above for abnormal recipient cells.

III. Patients who have undergone an allogeneic hematopoietic cell transplantation for whom donor and recipient cells cannot be determined:

Analysis: analyze 20 cells. As in case scenarios outlined here, follow guidelines for recipient cells as set forth above.

\subsubsection{FISH analysis}

6.3.2.1 Interphase FISH analysis may be used as a primary testing methodology in conjunction with G-banded chromosome analysis for the evaluation of hematological neoplasms. FISH studies may be indicated to (i) provide a rapid result to aid in the differential diagnosis or planning of therapy; (ii) detect a cryptic chromosomal abnormality or gene rearrangement, especially when G-banded chromosome analysis yields normal results; (iii) detect clinically significant gene amplification, which may also require metaphase FISH analysis to document the tandem nature of this rearrangement on the same chromosome or the presence of double minutes; (iv) provide an alternative diagnostic method when no metaphase cells are obtained by blood or bone marrow cultures; and (v) detect abnormalities in samples that are not adequate or not suitable for G-banded chromosome analysis.

6.3.2.2 Characterization of the initial diagnostic interphase FISH abnormal signal pattern is important and will allow future monitoring of the patient's disease.

6.3.2.3 Metaphase FISH analysis and/or sequential G-banded chromosome analysis to metaphase FISH analysis may be useful and provides a useful methodology to characterize variant chromosomal abnormalities or gene rearrangements as demonstrated by a variant abnormal interphase FISH signal pattern.

6.3.2.4 Analysis and documentation of FISH studies should be in accordance with Section E9 of these standards and guidelines for clinical genetics laboratories.

\subsubsection{CMA analysis}

6.3.3.1 CMA analysis can add valuable information that will support and supplement both G-banded chromosome analysis and FISH. It can detect small cryptic clinically significant copy number changes (CNCs) in various hematological neoplasms. Additionally, CMA SNP platforms can also detect copy-neutral loss of heterozygosity $(\mathrm{cnLOH})$. However, this technology cannot detect balanced chromosomal rearrangements.

6.3.3.2 The clinical utility of genome-wide CMAs in cancer diagnostics is growing rapidly. This technology is being used to better identify high-risk patients and predict clinical outcomes. In view of the rapid introduction of CMAs into clinical practice, it is important that laboratories stay up-to-date with this technology.

6.3.3.3 Analysis and documentation of CMA studies should be in accordance with Section E11 of these standards and guidelines for clinical genetics laboratories.

\subsubsection{Recommended cytogenetic analysis scheme in hematological neoplasms}

6.3.4.1 Acute leukemias

Bone marrow is the preferred specimen for acute leukemias, but peripheral blood can be used when $>10 \%$ circulating blast cells are present. ${ }^{8}$ Interphase FISH analysis performed on bone marrow smears or core biopsy touch imprints is an alternative in cases with a dry tap and/or hemodiluted bone marrow aspirate and absent/low circulating blast cells. A strong collaboration with the oncologist and pathologist is important for 
establishing the order of testing and additional tests that should be undertaken. ${ }^{9}$

1. AML

- G-banded chromosome analysis should preferably be performed first. However, interphase FISH analysis for KMT2A (MALL) gene rearrangement is highly recommended on all diagnostic AML samples because these abnormalities are often cryptic and have a pronounced prognostic impact.

- In case of a successful normal chromosome analysis with a clear diagnosis of AML by morphology and flow cytometry, additional interphase and metaphase FISH analyses are recommended to exclude cryptic rearrangements. Depending on the morphology and flow cytometry results, the following FISH probes can be added:

a. RUNX1-RUNX1T1 (AML1-ETO) fusion probes

b. $C B F B$ rearrangement or $C B F B-M Y H 11$ fusion probes: inv(16) and $\mathrm{t}(16 ; 16)$ resulting in $C B F B$ MYH11 fusion can be subtle in cases with suboptimal G-banded chromosomes quality

c. KMT2A $(M L L)$ rearrangement probes

d. PML-RARA fusion probes: PML-RARA fusion is diagnostic of acute promyelocytic leukemia (APL), which is usually strongly suspected at diagnosis based on the patient's presentation and blast cell morphology. A RARA break-apart probe can be used to detect variant translocations in which RARA fuses with a different partner

- In case of an incomplete/unsuccessful chromosome analysis or if the laboratory is unable to maintain a short TAT for chromosome analysis, then the following probes can be bundled in an AML FISH panel, which should be performed on the diagnostic specimen:

a. RUNX1-RUNX1T1 (AML1-ETO) fusion probes

b. $C B F B$ rearrangement or $C B F B-M Y H 11$ fusion probes

c. KMT2A $(M L L)$ rearrangement probes

d. $-5 / 5 q-$ probes

e. $-7 / 7 q-$ probes

f. PML-RARA fusion probes: if there is suspicion of APL based on the patient's presentation and blast cell morphology

- MECOM (EVI1) rearrangement probes should be considered when chromosome analysis is suggestive of $\operatorname{an} \operatorname{inv}(3)$ or $t(3 ; 3)$.

- $\quad$ Recent CMA studies revealed acquired CNCs and region of $\mathrm{cnLOH}$ that add independent prognostic impact in AML. CMA analysis can detect CNCs that are more specific to primary AML, whereas others are more specific to therapy-related AML. ${ }^{10}$ In addition, regions of $\mathrm{cnLOH}$ are more often detected in patients with normal karyotypes than with abnormal karyotypes. ${ }^{11,12}$

2. ALL

- B-lineage ALL is more frequent, accounting for $85 \%$ of pediatric ALL and $75 \%$ of adult ALL. ${ }^{1}$

- In pediatric/young adult B-lineage ALL, G-banded chromosome analysis should be performed simultaneously with interphase FISH analysis using a panel that includes the following probes:

a. BCR-ABL1 fusion probes

b. KMT2A $(M L L)$ rearrangement probes

c. ETV6-RUNX1 fusion probes: for ETV6-RUNX1 fusion, ETV6 deletion, and AAMP21 (intrachromosomal amplification of chromosome 21)

d. Centromeric probes for chromosomes 4 and 10: for trisomies of chromosomes 4 and 10

In adult B-lineage ALL, G-banded chromosome analysis should be performed simultaneously with interphase FISH analysis using the following probes:

a. BCR-ABL1 fusion probes

b. KMT2A $(M L L)$ rearrangement probes

- In both pediatric and adult B-lineage ALL, and depending on the blast cell morphology, flow cytometry, chromosome analysis, and FISH results, additional interphase FISH testing should be considered, including:

a. CRLF2 rearrangement probes: for $P 2 R Y 8$ CRLF2 fusion and IGH-CRFL2 fusion (Ph-like ALL) ${ }^{13}$

b. PDGFRB rearrangement probes (Ph-like ALL $)^{13}$

c. $C D K N 2 A / B(9 \mathrm{p} 21.3)$ probe: $9 \mathrm{p} 21.3$ deletion is common in both B- and T-lineage ALLs, but its prognostic significance has been debated; however, it provides a clonal target for future monitoring of the patient's disease in the absence of other FISH targets

d. PAX5 (9p13.2) probe

MYC rearrangement and/or IGH-MYC fusion probes should be considered in both pediatric and adult ALL, where the morphology and flow cytometry results are suggestive of B-cell ALL (Burkitt leukemia variant)

- In T-lineage ALL, G-banded chromosome analysis should be performed first. Interphase FISH analysis is optional and could include the following probes:

a. BCR-ABL1 fusion probes: for $B C R-A B L 1$ fusion and $A B L 1$ amplification

b. KMT2A $(M L L)$ rearrangement probes

In ALL, CMA analysis can be very helpful for detecting cryptic CNCs, with proven relevance to diagnosis, prognosis, and therapeutic response. ${ }^{14-16}$ Examples include deletions involving PAX5 and $I K Z F 1$ genes. It can also help clarify the structure of complex chromosomal rearrangements. Finally, 
CMA SNP platforms can detect whole-chromosome cnLOH due to "doubling" of a near-haploid or low hypodiploid clone, which manifests in the form of a hyperdiploid or near-triploid karyotype. The prognosis of these two entities is very different.

\subsubsection{Myelodysplastic syndromes}

Bone marrow is the preferred specimen for MDS. ${ }^{17}$ Interphase FISH analysis performed on bone marrow smears or core biopsy touch imprints is an alternative in cases with a dry tap and/or hemodiluted bone marrow aspirate. A strong collaboration with the oncologist and pathologist is important in MDS cases, where other nonneoplastic hematological disorders can have a similar presentation.

- G-banded chromosome analysis should preferably be performed first. In case of an incomplete/unsuccessful chromosome analysis or if the laboratory is unable to maintain a short TAT for chromosome analysis, the following probes can be bundled in an MDS FISH panel, ${ }^{18}$ which should be performed on the diagnostic specimen: a. $-5 / 5 \mathrm{q}$ - probes

b. $-7 / 7 \mathrm{q}$ - probes

c. Centromeric probe for chromosome 8 : for trisomy 8 d. 20q- probe

- Recent data suggest that MDS exhibits abundant clonal $\mathrm{CNCs}$ and $\mathrm{cnLOH}$, often in the setting of a normal metaphase karyotype and with no previously identified clonal markers. CMA analysis is proving to be very useful in uncovering these genomic aberrations in MDS. ${ }^{19,20}$ Examples include cryptic $5 \mathrm{q}$ deletions distal to the EGR1 gene (5q31). These can be missed by G-banded chromosome and FISH analyses. ${ }^{21}$

6.3.4.3 Myeloproliferative neoplasms and myelodysplastic syndromes/myeloproliferative neoplasms.

This is a heterogeneous group of clonal stem disorders that is broadly divided into three groups. ${ }^{9,22}$ The first is the classical MPN group, which includes CML (BCR-ABL1 fusion positive), polycythemia vera, essential thrombocythemia, primary myelofibrosis, chronic neutrophilic leukemia, chronic eosinophilic leukemia not otherwise specified, mastocytosis, and MPN unclassified. The second group includes myeloid and lymphoid neoplasms associated with eosinophilia and abnormalities of PDGFRA, PDGFRB, or FGFR1. The third is the MDS/MPN group, which includes chronic myelomonocytic leukemia, atypical CML ( $B C R-A B L 1$ fusion negative), juvenile myelomonocytic leukemia, and MDS/MPN unclassified.

\section{CML}

- Bone marrow is the preferred specimen for CML; however, peripheral blood may be used if the level of blasts is $>10 \%$.

- The $\mathrm{t}(9 ; 22)(\mathrm{q} 34 ; \mathrm{q} 11.2)$ is detectable in $90-95 \%$ of CML cases at diagnosis. The remaining $5-10 \%$ of cases have either a variant $t(9 ; 22)$ or a cryptic $B C R$ $A B L 1$ fusion undetectable by chromosome analysis.

- Therefore, both G-banded chromosome analysis as well as interphase FISH analysis using $B C R-A B L 1$ fusion probes should be performed simultaneously at diagnosis.

- It is important to establish whether additional chromosome abnormalities are present at diagnosis, including an additional der(22), i(17q), and trisomy 8 . These are warning signs that might be associated with inferior overall survival and increased risk of progression to accelerated phase. ${ }^{23,24}$

- The CML National Comprehensive Cancer Network $(\mathrm{NCCN})$ guidelines recommend that cytogenetic studies (both G-banded chromosome and BCR$A B L 1$ fusion FISH analyses) and quantitative RT-PCR $B C R-A B L 1$ fusion testing be performed at diagnosis. If no $B C R-A B L 1$ fusion can be detected, molecular testing for mutations associated with other myeloproliferative conditions is indicated.

2. Other MPNs

- Bone marrow is the preferred specimen for other MPNs; however, peripheral blood may be used if there is peripheral involvement. With few exceptions, cytogenetic abnormalities are usually not specific in other MPNs. Typical abnormalities of myeloid neoplasms are usually observed and can be useful in demonstrating evidence of clonality.

- Interphase FISH analysis performed on bone marrow smears or core biopsy touch imprints is an alternative in cases with a dry tap and/or hemodiluted bone marrow aspirate. A strong collaboration with the oncologist and pathologist is important.

- The exclusion of $B C R-A B L 1$ fusion is necessary for the differential diagnosis of other MPNs from CML.

- Other specific FISH probes recommended in other MPNs based on the pathology input include FIP1L1PDFGRA fusion, PDGFRB rearrangement, and FGFR1 rearrangement probes in myeloid/lymphoid neoplasms with eosinophilia. MPNs with these gene rearrangements can be treated with targeted therapies (i.e., tyrosine kinase inhibitors).

\subsubsection{Plasma cell dyscrasias}

- A bone marrow specimen is required for MM. For FISH and/or CMA analyses, plasma cell separation is recommended to enrich for the $\mathrm{CD} 138^{+}$plasma cell fraction in bone marrow samples with low plasma cell percentages (see Section 6.2.2.3). ${ }^{4,5}$

- G-banded chromosome analysis should be performed (as described above) simultaneously with interphase FISH analysis using a panel that includes the following probes: ${ }^{25-27}$ a. 1q21.3 probe (including CKS1B): for 1q21 copy gain, which has been linked to adverse prognosis 
b. $13 \mathrm{q} 14.2 \mathrm{q} 14.3$ probes (including $R B 1$ ): $13 \mathrm{q} 14.2 \mathrm{q} 14.3$ deletion is common in MM but, when detected only by FISH, it is not predictive of survival in the absence of other adverse cytogenetic abnormalities. However, it provides a clonal target for future monitoring of the patient's disease in the absence of other FISH targets. 13q deletion detected by G-banded chromosome analysis still retains its prognostic value

c. IGH rearrangement probes: if IGH is rearranged, including the classical gene disruption as well as deletion of either the $5^{\prime}$ or $3^{\prime}$ region of IGH, then reflex to IGH-FGFR3, IGH-CCNDI, and IGH-MAF fusion probes.

d. TP53 (17p13.1) probe

e. Probes for three of the odd-numbered chromosomes often trisomic in hyperdiploid MM (e.g., chromosomes $5,9,11,15$, and 19)

- The use of CMA analysis on the enriched plasma cell fraction has been shown to be very valuable in detecting clinically relevant $\mathrm{CNCs}{ }^{28-30}$

\subsubsection{Chronic lymphocytic leukemia}

- CLL is a mature B-cell neoplasm diagnosed by B-cell count, morphology, and flow cytometry. Cytogenetically, either peripheral blood or bone marrow can be used in CLL. G-banded chromosome analysis should be performed simultaneously with interphase FISH analysis. ${ }^{31}$ CLL cell stimulation in culture using CpGoligonucleotides greatly improves the detection rate of clonal cytogenetic abnormalities by G-banded chromosome analysis. ${ }^{6,7}$

- To assign the patient into clinically relevant prognostic subgroups, the following panel of FISH probes is recommended:
a. $\operatorname{ATM}(11 \mathrm{q} 22.3)$ probe
b. Centromeric probe for chromosome 12: for trisomy 12
c. $13 q 14.3$ probe (including D13S319)
d. TP53 (17p13.1) probe

- FISH can also be useful for the differential diagnosis with mantle cell lymphoma (MCL), for which FISH using the $I G H-C C N D 1$ fusion probes is recommended.

- In CLL, CMA analysis has proven to be very effective in detecting $\mathrm{CNCs}$ and $\mathrm{cnLOH}$ at genomic regions with established prognostic significance, and it provides a much higher resolution compared to G-banded chromosome and FISH analyses. ${ }^{32,33}$ Examples include 13q14 deletions, which are quite heterogeneous. ${ }^{34}$ Moreover, clinically relevant genomic alterations in CLL involve mostly deletions and duplications, whereas most balanced translocations are relatively rare and are of unclear significance.

\subsubsection{B- and T-cell lymphomas}

- For all lymphomas, the preferred tissue is lymph node or biopsy material from a suspected lymphoid mass. If fresh material is available, G-banded chromosome analysis is recommended.

- Interphase FISH analysis using relevant probes performed on lymph node tissue sections, fine needle aspirate smears, and/or touch imprints should be included.

- For lymph node cytogenetic analysis in lymphomas, see Section E6.5-6.8.

- Bone marrow or peripheral blood analysis will not detect clonal chromosomal abnormalities if there is no evidence of infiltration. For FISH analysis, bone marrow smears or core biopsy touch imprints can be used.

\subsection{TAT AND REPORTING}

\subsubsection{TAT}

6.4.1.1 Specific chromosomal abnormalities are crucial for establishing a diagnosis and have direct relevance to specific treatment. Therefore, an effort should be made to expedite communicating the cytogenetic analyses results to the oncologist. It is recommended that the cytogenetics laboratory should have a written policy describing how cases are prioritized in the laboratory.

\subsubsection{TAT guidance:}

a. Initial diagnostic workup: It is strongly recommended that the preliminary result should be reported within 7 calendar days, and the final results should be reported within 21 calendar days.

b. Follow-up studies: It is strongly recommended that the final results should be reported within 21 calendar days.

c. FISH studies: Reporting the FISH results within 3-5 working days from the time of receiving the specimen is recommended whenever possible.

\subsubsection{Reporting}

6.4.2.1 The most recent edition of the International System for Human Cytogenetic Nomenclature (ISCN) should be used to report the cytogenetics results. ${ }^{35}$

6.4.2.2 The number of cells analyzed (both normal and abnormal) should be documented in the final report.

6.4.2.3 For CMA analysis, clones and subclones cannot be ascertained with certainty; however, the percentage of cells (levels of mosaicism) can be provided to give an estimate of possible clones/subclones and clonal diversity.

6.4.2.4 If a potential nonmosaic constitutional abnormality is observed, analysis of a PHA-stimulated peripheral blood sample during remission is strongly recommended to confirm that the abnormality is constitutional and not clonal.

6.4.2.5 At the time of initial diagnosis, finding a single abnormal metaphase cell, even one that is potentially significant, cannot be used as evidence of clonality unless there is strong supporting evidence of clonality for the same abnormality by either FISH or other molecular technique.

6.4.2.6 The final cytogenetic report of hematological acquired chromosomal abnormalities should contain the following information: 
1. Patient identification using two different identifiers

2. Patient medical record number and/or laboratory identification number

3. Referring physician

4. Sample information (type, date of withdrawal and receipt, and date of report)

5. Reason for referral or suspected diagnosis

6. ISCN nomenclature of cytogenetic studies performed

7. Narrative description of the abnormalities observed, including modal chromosome number in each clone (to the extent possible), and numerical and structural abnormalities. The report should comment on the clinical significance of the abnormalities observed, including clinically relevant genes involved, possible disease association, and prognostic significance.

8. Literature references to support the clinical interpretation and to provide helpful information for the oncologist.

\section{DISCLOSURE}

All of the authors direct clinical cytogenetics laboratories that run the tests discussed in the current standards and guidelines on a fee-for-service basis.

\section{REFERENCES}

1. Swerdlow SH, Campo E, Harris NL, Jaffe ES, Pileri SA, Stein H, Thiele J, Vardiman JW (eds). WHO Classification of Tumours: WHO Classification of Tumours of Haematopoietic and Lymphoid Tissues 2008 (2008). 4th edn. IARC Press: Lyon, France.

2. Mitelman F, Johansson B and Mertens F (eds). Mitelman Database of Chromosome Aberrations and Gene Fusions in Cancer (2015). http://cgap.nci. nih.gov/Chromosomes/Mitelman.

3. Hernández JM, Gutiérrez NC, Almeida J, et al. IL-4 improves the detection of cytogenetic abnormalities in multiple myeloma and increases the proportion of clonally abnormal metaphases. BrJ Haemato/ 1998;103:163-167.

4. Stevens-Kroef M, Weghuis DO, Croockewit S, et al. High detection rate of clinically relevant genomic abnormalities in plasma cells enriched from patients with multiple myeloma. Genes Chromosomes Cancer 2012;51:997-1006.

5. Shin SY, Jang S, Park CJ, et al. Application of an immune-magnetic cell sorting method for CD138-positive plasma cells in FISH analysis of multiple myeloma. Int J Lab Hemato/ 2012;34:541-546.

6. Heerema NA, Byrd JC, Dal Cin PS, et al.; Chronic Lymphocytic Leukemia Research Consortium. Stimulation of chronic lymphocytic leukemia cells with CpG oligodeoxynucleotide gives consistent karyotypic results among laboratories: a CLL Research Consortium (CRC) Study. Cancer Genet Cytogenet 2010;203:134-140.

7. Shi M, Cipollini MJ, Crowley-Bish PA, Higgins AW, Yu H, Miron PM. Improved detection rate of cytogenetic abnormalities in chronic lymphocytic leukemia and other mature B-cell neoplasms with use of CpG-oligonucleotide DSP30 and interleukin 2 stimulation. Am J Clin Pathol 2013;139:662-669.

8. Weinkauff R, Estey EH, Starostik P, et al. Use of peripheral blood blasts vs bone marrow blasts for diagnosis of acute leukemia. Am J Clin Pathol 1999;111: 733-740.

9. Vardiman JW, Thiele J, Arber DA, et al. The 2008 revision of the World Health Organization (WHO) classification of myeloid neoplasms and acute leukemia: rationale and important changes. Blood 2009;114:937-951.

10. Itzhar N, Dessen P, Toujani S, et al. Chromosomal minimal critical regions in therapy-related leukemia appear different from those of de novo leukemia by high-resolution aCGH. PLoS One 2011;6:e16623.

11. Walter MJ, Payton JE, Ries RE, et al. Acquired copy number alterations in adult acute myeloid leukemia genomes. Proc Natl Acad Sci USA 2009;106: 12950-12955.

12. Parkin $B$, Erba $H$, Ouillette $P$, et al. Acquired genomic copy number aberrations and survival in adult acute myelogenous leukemia. Blood 2010;116: 4958-4967.
13. Roberts KG, Li Y, Payne-Turner $D$, et al. Targetable kinase-activating lesions in Ph-like acute lymphoblastic leukemia. N Engl J Med 2014;371:1005-1015.

14. Collins-Underwood JR, Mullighan CG. Genomic profiling of high-risk acute lymphoblastic leukemia. Leukemia 2010;24:1676-1685.

15. Simons A, Stevens-Kroef M, El Idrissi-Zaynoun N, et al. Microarray-based genomic profiling as a diagnostic tool in acute lymphoblastic leukemia. Genes Chromosomes Cancer 2011;50:969-981.

16. Baughn LB, Biegel JA, South ST, et al. Integration of cytogenomic data for furthering the characterization of pediatric B-cell acute lymphoblastic leukemia: a multi-institution, multi-platform microarray study. Cancer Genet 2015;208: $1-18$.

17. Cherry AM, Slovak ML, Campbell LJ, et al. Will a peripheral blood (PB) sample yield the same diagnostic and prognostic cytogenetic data as the concomitant bone marrow (BM) in myelodysplasia? Leuk Res 2012;36:832-840.

18. Greenberg PL, Tuechler H, Schanz J, et al. Revised international prognostic scoring system for myelodysplastic syndromes. Blood 2012;120: 2454-2465.

19. Heinrichs S, Kulkarni RV, Bueso-Ramos CE, et al. Accurate detection of uniparental disomy and microdeletions by SNP array analysis in myelodysplastic syndromes with normal cytogenetics. Leukemia 2009;23:1605-1613.

20. Thiel A, Beier M, Ingenhag D, et al. Comprehensive array CGH of normal karyotype myelodysplastic syndromes reveals hidden recurrent and individual genomic copy number alterations with prognostic relevance. Leukemia 2011;25:387-399.

21. MacKinnon RN, Kannourakis G, Wall M, Campbell LJ. A cryptic deletion in 5 q31.2 provides further evidence for a minimally deleted region in myelodysplastic syndromes. Cancer Genet 2011;204:187-194.

22. Vardiman J, Hyjek E. World health organization classification, evaluation, and genetics of the myeloproliferative neoplasm variants. Hematology Am Soc Hematol Educ Program 2011;2011:250-256.

23. Palandri F, Testoni N, Luatti S, et al. Influence of additional cytogenetic abnormalities on the response and survival in late chronic phase chronic myeloid leukemia patients treated with imatinib: long-term results. Leuk Lymphoma 2009;50:114-118.

24. Fabarius A, Leitner A, Hochhaus A, et al.; Schweizerische Arbeitsgemeinschaft für Klinische Krebsforschung (SAKK) and the German CML Study Group. Impact of additional cytogenetic aberrations at diagnosis on prognosis of CML: longterm observation of 1151 patients from the randomized CML Study IV. Blood 2011:118:6760-6768.

25. Dimopoulos M, Kyle R, Fermand JP, et al.; International Myeloma Workshop Consensus Panel 3. Consensus recommendations for standard investigative workup: report of the International Myeloma Workshop Consensus Panel 3. Blood 2011;117:4701-4705.

26. Munshi NC, Anderson KC, Bergsagel PL, et al.; International Myeloma Workshop Consensus Panel 2. Consensus recommendations for risk stratification in multiple myeloma: report of the International Myeloma Workshop Consensus Panel 2. Blood 2011;117:4696-4700.

27. van de Donk NW, Sonneveld P. Diagnosis and risk stratification in multiple myeloma. Hematol Oncol Clin North Am 2014;28:791-813.

28. Agnelli L, Mosca L, Fabris S, et al. A SNP microarray and FISH-based procedure to detect allelic imbalances in multiple myeloma: an integrated genomics approach reveals a wide gene dosage effect. Genes Chromosomes Cancer 2009;48:603-614

29. Avet-Loiseau H, Li C, Magrangeas F, et al. Prognostic significance of copynumber alterations in multiple myeloma. J Clin Oncol 2009;27:4585-4590.

30. Walker $\mathrm{BA}$, Leone $\mathrm{PE}$, Chiecchio L, et al. A compendium of myeloma-associated chromosomal copy number abnormalities and their prognostic value. Blood 2010;116:e56-e65.

31. Zenz T, Mertens D, Döhner H, Stilgenbauer S. Importance of genetics in chronic lymphocytic leukemia. Blood Rev 2011;25:131-137.

32. Hagenkord JM, Monzon FA, Kash SF, Lilleberg S, Xie Q, Kant JA. Array-based karyotyping for prognostic assessment in chronic lymphocytic leukemia: performance comparison of Affymetrix 10K2.0, 250K Nsp, and SNP6.0 arrays. J Mol Diagn 2010;12:184-196.

33. O'Malley DP, Giudice C, Chang AS, et al. Comparison of array comparative genomic hybridization ( $\mathrm{aCGH}$ ) to FISH and cytogenetics in prognostic evaluation of chronic lymphocytic leukemia. Int J Lab Hematol 2011;33:238-244.

34. Ouillette P, Collins R, Shakhan $S$, et al. The prognostic significance of various $13 q 14$ deletions in chronic lymphocytic leukemia. Clin Cancer Res 2011;17:6778-6790

35. Shaffer LG, McGowan-Jordan J, Schmid M (eds). An International System for Human Cytogenetic Nomenclature (ISCN) 2013. S. Karger: Basel, Switzerland. 